\title{
Comparison of Tympanic and Tail Temperatures in Angus and Brahman
}

\section{Steers}

\author{
Serdal Dikmen ${ }^{1}$, Kaitlyn M Sarlo Davila², Eduardo Rodriquez², Tracy L Scheffler ${ }^{2}$, Pascal A Oltenacu and Raluca G Mateescu ${ }^{2, *}$ \\ ${ }^{1}$ Department of Animal Science, Faculty of Veterinary Medicine, Bursa Uludağ University, Bursa, Turkey \\ ${ }^{2}$ Department of Animal Sciences, University of Florida, Gainesville, USA
}

*Corresponding author: Raluca G Mateescu, Department of Animal Sciences, University of Florida, Gainesville, USA, E-mail: raluca@ufl.edu

Received: 09 Nov, 2020 | Accepted: 23 Nov, 2020 | Published: 27 Nov, 2020

Citation: Dikmen S, Davila KMS, Rodriquez E, Scheffler TL, Oltenacu PA, et al. (2020) Comparison of Tympanic and Tail Temperatures in Angus and Brahman Steers. J Anim Sci Res 5(1): dx.doi.org/10.16966/2576-6457.147

Copyright: (C) 2020 Dikmen S, et al. This is an open-access article distributed under the terms of the Creative Commons Attribution License, which permits unrestricted use, distribution, and reproduction in any medium, provided the original author and source are credited.

\begin{abstract}
In cattle, core body temperature can be used as an important indicator of heat stress level. However, accurately recording core body temperature can be difficult and labor intensive. The objectives of the current study were 1) to compare the recorded tympanic and tail body temperature measurements in steers and 2) to determine the body temperature change of Angus and Brahman steers in a hot and humid environment. Data was analyzed using a repeated measure model where repeated measures were hourly tympanic and tail temperatures and their difference for individual steers during the day of the experiment. There was a significant breed effect $(P=0.01)$, hour $(P<0.0001)$ and breed by hour interaction $(P<0.0001)$ for the tympanic temperature. Brahman steers, which are known to have superior thermotolerance, maintained a lower body temperature than the Angus steers during the afternoon under grazing conditions. In the Brahman steers there was only a minimal increase in the body temperature throughout the day, an evidence of the thermotolerance ability of the breed. In the Angus steers, which experienced an increase in their body temperature from hour to hour with a peak around 1600 hour; there was a significant difference between the tympanic and tail temperature during the times when the body temperature as measured by the tympanic recordings was the highest (1300 to 1700 hour). Our results indicate that the tympanic temperature can be used to accurately and continuously monitor core body temperature in a natural environment for up to several days and without disturbing the animal.
\end{abstract}

Keywords: Heat Stress; Thermotolerance; Angus; Brahman

\section{Introduction}

Body temperature can be used as an indicator of thermotolerance ability in cattle and is the most common parameter to determine cattle's response to the environment. Body temperature is a result of physiological and chemical processes in the body which are also related to production traits in cattle [1]. Body temperature changes as a result of environmental or other conditions and these changes in body temperature affect the appetite, production, and reproduction level in cattle [2]. The ability to monitor core body temperature is important for many aspects of biological and physiological research related to thermotolerance, however, it can be difficult to perform in field conditions. Besides being labor intensive, animals need to be restrained during the process. This presents additional challenges and expenses especially for beef cattle which are reared on pasture. Furthermore, restraining can cause stress and an increase in body temperature which results in inaccurate data. Recently, data loggers have been used for monitoring internal temperature continuously: HOBO Water Temperature Pro v2 (Model U22-001, Onset Company, Bourne, MA with an accuracy of $\pm 0.21^{\circ} \mathrm{C}$ at a resolution of $0.02^{\circ} \mathrm{C}$ ) and iButton data loggers $(1922 \mathrm{~L}, 1922 \mathrm{~T}$ or $1925 \mathrm{~L}$, Maxim Integrated, San Jose, CA; accuracy of $\pm 0.0625^{\circ} \mathrm{C}$ at 11 -bit resolution [3,4]. For heifers or cows, these data loggers can be used vaginally to record the body temperature continuously for several days by attaching the data loggers to a blank Controlled Internal Drug Release (CIDR, Pfizer Animal Health, New York, NY). Keeping the CIDRs for longer than 5 days is not recommended because tehy might cause irritation and infection and the body temperature might change slightly, which can affect the result of the experiment. The use of data loggers intravaginally under correct conditions provides accurate continuous data of core body temperature. However, this type of data collection is restricted to female animals. In addition to vaginal temperature, body temperature can be measured internally or externally on different locations of an animal, such as rectum, ear, reticulorumen and on the forehead or udder surface. Common locations for core body temperature in cattle are the rectum, ear canal, rumen and vagina [5]. Temperature recorded in the ear canal is a very reliable indicator of internal core body temperature because the blood flowing through the vessels of the tympanic membrane comes from the branch of the carotid artery that also supplies the hypothalamus [6]. In a study comparing the ear canal, lumen of the rectum and the rumen, [7] found no difference between the tympanic and rectal temperatures, while the rumen temperature was higher. One drawback of the tympanic temperature is the possibility of ear infections if recording is attempted over multiple days. Another 
option for continuous measurement of core body temperature over several days without disturbing the animal is using data loggers under the tail, however, there is very little information to date on the comparison of tympanic and tail body temperatures of beef cattle.

Body temperature is affected by many factors, including breed, health or physiological status (sickness, heat, gestation, or stage of lactation), environmental humidity and temperature, feed consumption level, feed ingredients, age, BCS, coat color, and season [8]. In beef cattle on pasture, body temperature is lowest between $0700-0800$ hours $\left(38.2^{\circ} \mathrm{C}\right)$ and reaches the peak $\left(39.6^{\circ} \mathrm{C}\right)$ at 1600 hour during the day [9]. Brahman and Angus cattle have previously been reported to exhibit significantly different body temperatures under various heat stress conditions $[4,10]$.

The objectives of this study were 1) to compare the recorded tympanic and tail body temperature measurements in steers and 2) to determine the body temperature change of Angus and Brahman steers in a hot and humid environment.

\section{Material and Methods}

\section{Animals and data collection}

Animal use was approved by the University of Florida Institutional Animal Care and Use Committee (Protocol No. 201910571).

The study was conducted at the University of Florida Beef Teaching Unit (Gainesville, Florida). Angus ( $\mathrm{n}=13)$ and Brahman $(\mathrm{n}=11)$ steers were used to determine the change in body temperature during one day while grazing on pasture. Body temperature was measured in the ear (Figure 1A) as tympanic temperature and under the tail (Figure 1B) as tail temperature using iButton data loggers (model 1922L; Maxim Integrated, San Jose, CA, USA). All iButtons were calibrated before the start of the experiment. The iButtons and a Hobo data logger (model: U22) set to record every 5 min were placed in an incubator at a temperature of $39^{\circ} \mathrm{C}$ for 2 hours. The iButton temperature recordings were compared with Hobo data logger and this difference was used to calibrate each iButton. To measure the tympanic temperature, the iButtons were placed in the right ear and the ear canal was filled with cotton, then the ear was taped with self-adhesive bandages (VetWrap, 3M Corporation, St. Paul, MN). A string was attached to each iButton to facilitate recovery. For the tail temperature measurement, the iButtons were placed under the tail, covered with cotton and taped with self-adhesive bandages. Tympanic and tail temperatures of the steers were measured every $15 \mathrm{~min}$ from 0600 hour May 21, 2019, to 1830 hour May 21, 2019. Animals with only one type of temperature available at the end of the experiment (tympanic or tail) were removed from the analysis. The final dataset had 10 Angus and 8 Brahman animals with complete tympanic and tail temperatures across the entire day.

During the study, environmental variables of dry bulb temperature, relative humidity, dew point, temperature and black globe temperature were recorded near the pasture every $15 \mathrm{~min}$. The Dry Bulb Temperature (DBT), Relative Humidity (RH) and dew point temperature were measured using the HOBO-U23 data logger (Onset Computer Corp., Bourne, MA) and black globe temperature was measured by using HOBO-U22 data logger. The temperature-humidity index (THI) was calculated using the following formula:

$$
\mathrm{THI}=(1.8 \times \mathrm{DBT}+32)-((0.55-0.0055 \times \mathrm{RH}) \times(1.8 \times \mathrm{DBT}-26))
$$

Where DBT=Dry Bulb Temperature $\left({ }^{\circ} \mathrm{C}\right)$ and $\mathrm{RH}=$ Relative Humidity (percent)

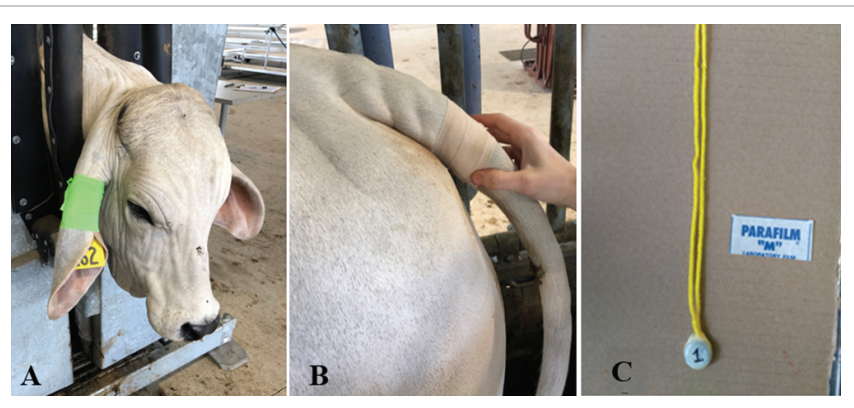

Figure 1: Tympanic (A) and tail (B) temperature data collection using iButton datalogger $(C)$ covered with parafilm in Brahman steer.

\section{Statistical analysis}

To assess how the tympanic temperature, the tail temperature and the difference between tympanic and tail temperatures for the two breed groups respond to varying intensity and duration of heat stress during the experiment, the temperature data was analyzed using a repeated measure model where repeated measures were hourly tympanic and tail temperatures and their difference for individual steers during the day of the experiment.

A likelihood ratio $\chi^{2}$ statistic was used to select the appropriate covariance structure of residuals and first order autoregressive covariance structure, AR (1), provided the best fit.

Data were analyzed with the MIXED procedure of SAS 9.4 (SAS Inst. Inc., Cary, NC) and REML estimation. The model included breed, hour and breed by hour interaction as fixed effects. The repeated effect was hour and REPEATED statement used SUBJECT=steer (breed), type $=\mathrm{AR}(1)$. The observations were hourly tympanic and tail temperatures and their difference for each steer. Least squares means were separated using LSMEANS with the PDIFF option.

\section{Results and Discussion}

The environmental conditions described by hourly dry bulb temperatures $\left({ }^{\circ} \mathrm{C}\right)$, relative humidity $(\%)$ and THI are shown in table 1. Heat stress ambient conditions based on THI can be categorized as: normal $\leq 74$; moderate 75-78; severe 79-83; very severe (emergency) $\geq 84$ (LWSI, 1970). In our study, cattle were exposed to normal conditions between 0700 and 0800 hours, moderate heat stress between 0800 and 1000 hours and severe heat stress between 1000 and 1900 hours with the maximum THI reaching 81.34 at 1500 hour.

\section{Breed effect on body temperature}

The least square means and standard errors for the tympanic and tail temperatures of Angus and Brahman steers are presented in figure 2. There was a significant breed effect $(\mathrm{P}=0.01)$, hour $(\mathrm{P}<0.0001)$ and breed by hour interaction $(\mathrm{P}<0.0001)$ for the tympanic temperature. For the tail temperature, only the hour effect $(\mathrm{P}<0.0001)$ was significant. The least square means for the tympanic temperature in Angus $\left(38.42^{\circ} \mathrm{C} \pm 0.10\right)$ and Brahman steers $\left(37.99^{\circ} \mathrm{C} \pm 0.11\right)$ were significantly different $(\mathrm{P}=0.008)$ and, the least square means for the tail temperature in Angus $\left(38.28^{\circ} \mathrm{C} \pm 0.12\right)$ and Brahman steers $\left(38.01^{\circ} \mathrm{C} \pm\right.$ $0.14)$ were not significantly different $(\mathrm{P}=0.16)$.

For both breeds, the lowest body temperature was recorded at 0900 hour when the least square means for tympanic and tail temperature for the Angus steers were $37.73^{\circ} \mathrm{C}$ and $37.85^{\circ} \mathrm{C}$, respectively, and for the Brahman steers were $37.58^{\circ} \mathrm{C}$ and $37.67^{\circ} \mathrm{C}$, respectively. The body 


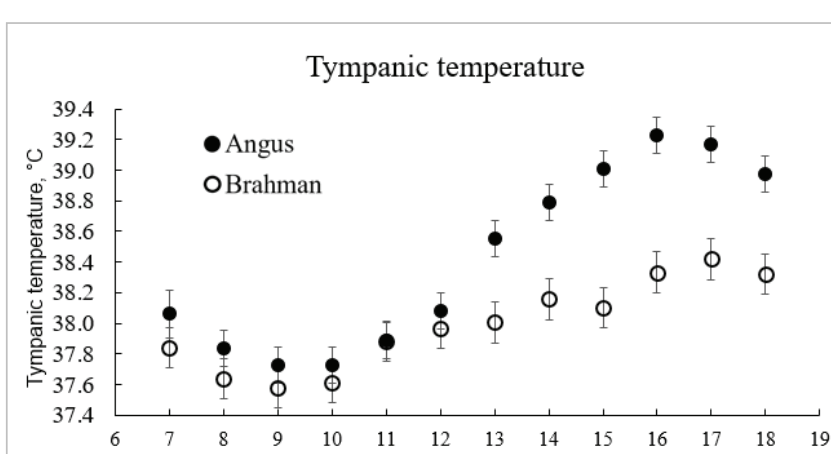

Tail temperature

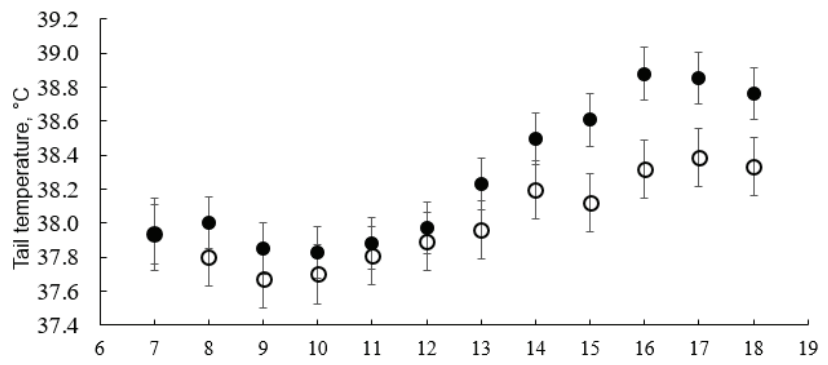

Figure 2: Least square means and standard errors for hourly average tympanic (top graph) and tail temperature (bottom graph) of Angus (closed circle) and Brahman (open circles) steers.

temperature increased throughout the day with the peak temperature occurring at $1600 \mathrm{~h}$ for Angus $\left(39.22^{\circ} \mathrm{C}\right.$ tympanic and $38.88^{\circ} \mathrm{C}$ tail) and $1700 \mathrm{~h}$ for Brahman steers $\left(38.42^{\circ} \mathrm{C}\right.$ tympanic and $38.39^{\circ} \mathrm{C}$ tail). Brahman steers, which are known to have superior thermotolerance, maintained a lower body temperature than the Angus steers during the afternoon under grazing conditions.

The body temperature change of both Brahman and Angus steers were consistent with the previous studies [3]. The time for the low and high body temperature is similar to that reported in the University of Florida multi breed population [4] where the lowest temperatures were recorded around 0700 and 0800 hours and peaked between 1600 and 2100 hour each day for both breed groups. Similar patterns have been described in crossbred Angus $\times$ Simmental steers under heat stress conditions where the average body temperature was lowest between 0700-0800 hour with a peak at 1700 hour followed by a significant reduction to $38.9^{\circ} \mathrm{C}$ by 1900 hour [11].

\section{Tympanic versus tail body temperature}

To compare the tympanic and tail measurement of temperature, the two measurements made on each steer each hour were paired. The least square means and standard errors for the hourly differences for the two breeds are shown in figure 3.

When analyzing the difference between tympanic and tail measures, because the two measures are not independent, the error variance of this difference is expected to be equal to the sum of the tympanic and tail variances minus twice the tympanic-tail covariance $\left(\sigma^{2}\right.$ ear $+\sigma^{2}$ tail $-2 \sigma$ ear, tail). The residual variance of the difference $(0.29 \pm 0.06)$ was smaller than the sum of the residual variances for the two individual measures $(0.37 \pm 0.03)$, as it accounts for the positive covariance between the two measures. The covariance structure of the residuals for the difference estimated by AR (1) parameter $(\rho)$ is similar with covariance structure of individual measures.
The least square means for the difference between the typanic and tail temperature for Angus $(0.143 \pm 0.14)$ and Brahman $(-0.023$ $\pm 0.15)$ were not significantly different from zero, consequently the breed effect was not significant $(\mathrm{P}=0.43)$. The hour effect was also not significant $(\mathrm{P}=0.18)$.

The least square means for the difference between tympanic and tail temperature for breed by hour interaction are shown in table 1 . In the Brahman steers, these estimates were not significantly different from zero throughout the hours of the study. However, in the Angus steers, which experienced a larger increase in their body temperature throughout the day with a peak around $1600 \mathrm{~h}$, this difference was large enough to be significantly different from zero during the times when the body temperature as measured by the tympanic recordings was the highest ( $1300 \mathrm{~h}$ to $1700 \mathrm{~h}$ ). This could provide an explanation for why there was no difference between the tympanic and tail temperature in the Brahman steers whose body temperature did not fluctuate much

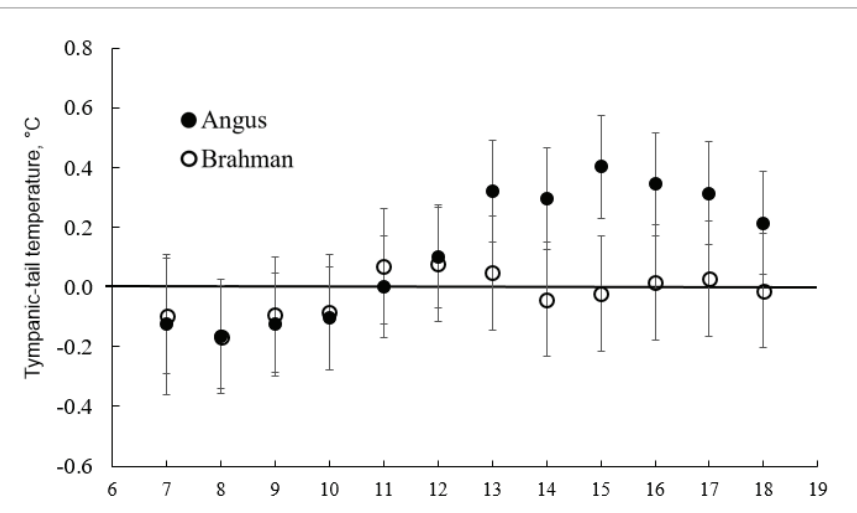

Figure 3: Least square means and standard errors for the difference between hourly average tympanic and tail temperature for Angus (closed circle) and Brahman (open circles) steers.

Table 1: The environmental variables of dry bulb temperature (DBT, $\left.{ }^{\circ} \mathrm{C}\right)$, relative humidity $(\mathrm{RH}, \%)$, and temperature humidity index (THI) during the experiment along with the Least Square means and the $P>|t|$ of the difference between tympanic and tail temperature for the breed by hour effect.

\begin{tabular}{|c|c|c|c|c|c|c|c|}
\hline & & & & \multicolumn{2}{|c|}{ Angus } & \multicolumn{2}{c|}{ Brahman } \\
\hline Hour & DBT & RH & THI & LS Means & P> |t I & LS Means & P>|t I \\
\hline 7 & 23.38 & 86.29 & 72.86 & 0.125 & 0.59 & -0.097 & 0.62 \\
\hline 8 & 25.37 & 79.83 & 75.45 & -0.167 & 0.34 & -0.166 & 0.39 \\
\hline 9 & 27.27 & 73.19 & 77.67 & -0.126 & 0.47 & -0.093 & 0.63 \\
\hline 10 & 29.33 & 64.55 & 79.56 & -0.105 & 0.54 & -0.085 & 0.66 \\
\hline 11 & 30.67 & 56.86 & 80.28 & 0.001 & 0.99 & 0.069 & 0.72 \\
\hline 12 & 32.24 & 50.29 & 81.27 & 0.102 & 0.55 & 0.076 & 0.69 \\
\hline 13 & 33.31 & 39.53 & 80.66 & 0.321 & 0.07 & 0.047 & 0.81 \\
\hline 14 & 33.21 & 41.63 & 80.93 & 0.296 & 0.09 & -0.042 & 0.83 \\
\hline 15 & 33.87 & 39.57 & 81.34 & 0.402 & 0.02 & -0.022 & 0.91 \\
\hline 16 & 34.31 & 35.21 & 81.01 & 0.344 & 0.05 & 0.016 & 0.93 \\
\hline 17 & 34.52 & 33.85 & 80.99 & 0.313 & 0.08 & 0.028 & 0.89 \\
\hline 18 & 34.16 & 37.30 & 81.22 & 0.214 & 0.22 & -0.012 & 0.95 \\
\hline
\end{tabular}


during the day. This also suggests that under normal environmental conditions and during periods when the body temperature is not expected to be elevated, both types of measurements are suitable and should allow for similar estimates. However, in experiments where body temperature is expected to be slightly elevated, which might be the case for the majority of the studies aimed at assessing the effect of heat stress, the tail temperatures might not allow for precise measurements and will tend to underestimate the body temperature. It is also important to note the approximately one-hour delay in the peak temperature recorded in Brahman compared to the Angus steers. This has been previously shown in a similar population of Brahman and Angus heifers from UF [4].

\section{Conclusions}

Our results confirm the sensitivity of Angus cattle to environmental THI while the Brahman cattle showed a relatively constant body temperature throughout the study. Our study showed that tympanic temperature is higher than tail temperature in steers reared under grazing conditions when THI is high. This difference was observed only in animals which respond to this higher THI by a large increase in their body temperature. While the tail temperature is easy to perform and can be used to monitor the body temperature of steers continuously, it is not an accurate measure of body temperature as it fails to accurately capture the increase in core body temperature. Tympanic temperature is a relatively easy measurement to perform and allows for a continuous measurement of body temperature in natural environment for up to several days and without disturbing the animal. In addition, the tympanic temperature describes the core body temperature more accurately illustrated by smaller residual variance relative to tail temperature measurement and the significantly higher difference between the two measures during heat stress hours.

\section{Applications}

Tympanic and tail body temperature measurements were compared in Angus and Brahman. Tympanic temperature was higher than tail temperature in grazing steers. Tympanic temperature is a relatively easy measurement to perform and allows for a continuous measurement of body temperature in natural environment for up to several days and without disturbing the animal.

\section{Acknowledgements}

The authors would like to thank the staff at the Beef Teaching Unit of the University of Florida.

\section{Declaration of Interest}

There is no conflict of interest for any of the authors that may affect their ability to objectively present research or data.

\section{Financial Support Statement}

This research was supported by USDA-NIFA Grant \#2017-6700726143 and Florida Agricultural Experiment Station Hatch FLAANS-005548. Serdal Dikmen was supported by TUBITAK-BIDEB Grant \#2219.

\section{References}

1. Hansen PJ (2004) Physiological and cellular adaptations of zebu cattle to thermal stress. Anim Reprod Sci 82-83: 349-360.

2. St-Pierre NR, Cobanov B, Schnitkey G (2003) Economic Losses from Heat Stress by US Livestock Industries. J Dairy Sci 86: E52-E77.

3. Dikmen S, Mateescu RG, Elzo MA, Hansen PJ (2018) Determination of the optimum contribution of Brahman genetics in an AngusBrahman multibreed herd for regulation of body temperature during hot weather. J Anim Sci 96: 2175-2183.

4. Mateescu RG, Sarlo-Davila KM, Dikmen S, Rodriguez E, Oltenacu PA (2020) The effect of Brahman genes on body temperature plasticity of heifers on pasture under heat stress. J Anim Sci 1-9.

5. Godyń D, Herbut P, Angrecka S (2019) Measurements of peripheral and deep body temperature in cattle-A review. J Therm Biol 79: 4249.

6. Bergen RD, Kennedy AD (2000) Relationship between vaginal and tympanic membrane temperature in beef heifers. Can J Anim Sci 80: 515-518.

7. Prendiville DJ, Lowe J, Earley B, Spahr C, Kettlewell P, et al (2002) Radiotelemetry Systems for Measuring Body Temperature. Beef Prod Ser No 57: 1-14.

8. Beatty DT, Barnes A, Taylor E, Pethick D, McCarthy M, et al. (2006) Physiological responses of Bos taurus and Bos indicus cattle to prolonged, continuous heat and humidity. J Anim Sci 84: 972-985.

9. Dikmen S, Alava E, Pontes E, Fear JM, Dikmen BY, et al. (2008) Differences in Thermoregulatory Ability Between Slick-Haired and Wild-Type Lactating Holstein Cows in Response to Acute Heat Stress. J Dairy Sci 91: 3395-3402.

10. Kaitlyn M Sarlo Davila, Heather Hamblen, Peter J Hansen, Serdal Dikmen, Pascal A Oltenacu, et al. (2019) Genetic parameters for hair characteristics and core body temperature in a multibreed Brahman-Angus herd. J Anim Sci 97: 3246-3252.

11. Sebastian H Scharf, Claudia Liebl, Elisabeth B Binder, Mathias V Schmidt, Marianne B Müller (2011) Expression and Regulation of the Fkbp5 Gene in the Adult Mouse Brain. PLoS ONE 6. 